\title{
Beratung und Entscheidung im Kontext kollektiven Ausagierens
}

(Deliberation in the context of collective acting out)

Alice PeChrigGL, KLAGENFURT

The paper is dealing with the philosophical implications of psychoanalytical work with and about groups. As a central psychoanalytical category, conflict emerges where the integration of dissident parts of the soul fails. In this general acceptation conflict is acting out or at least is conflict (or the oppressed part) tending to be acted out. As Freuds model for the topic was a socio-cultural one (Instanzen, Zensor, Es, Ich, Über-Ich, Idealich, Widerstand etc.) it can be revisited from a group analytical perspective in view of a reconceptualization of cultural and political conflict. In such a perspective the collective acting out of dissident parts is not the opposite of reasonable and deliberative action (Handeln), but part and parcel of such an action. It can even be seen as the motor for democratic participation not only as instituating power of movements but also as explicit figuration of the political "city" through deliberation of the citizens.

\section{Das begriffliche Spannungsfeld}

Egal mit welcher Schule, Zugangsweise oder Richtung wir es zu tun haben: Der herkömmliche philosophische Handlungsbegriff geht von bewussten oder zumindest bewusstseinsfähigen Handlungen, Taten oder Akten aus. Die Frage nach den Implikationen des psychoanalytischen Begriffs des Agierens für den philosophischen Handlungsbegriff gebietet dagegen eine radikale Erweiterung des Spannungsfeldes, dessen Grenzen hier mit den Termini "Agieren und Handeln" markiert sind. Für Freud ist Agieren der Effekt eines Vorstellens/Wünschens/Tuns im Register des zumeist unbewussten Lustprinzips; das neurotische Moment besteht dabei vornehmlich darin, dass dieses Prinzip für das Bewusstsein des Subjekts selbst wie auch für die anderen vermittels einer Rationalisierung verschleiert wird, sodass die Aktion bzw. das Agieren als realistisch und funktional erscheint: Die kapitalistische Unternehmerin etwa, die ihren unbewussten und durchaus antisozialen Bereicherungswünschen auf Kosten anderer 
frönt, wird ihr Agieren als rationales Handeln im Sinne einer ohnehin sich selbst regulierenden neoliberalen Gesellschaft darstellen, ja verkaufen; der Politiker, der seinen narzisstischen Beherrschungs- und Kontrollgelüsten frönt, führt rationalisierend oder ideologisch gesprochen seine Schafe zu ihrem Besten in die beste aller Welten. So lange die AkteurInnen ihre Motive und das Ganze ihres Agierens sich selbst und den anderen verschleiern, muss die politische Kosmetik im Vordergrund stehen und das Handeln verschwindet tendenziell hinter dem Agieren; die politische Macht wird privat angeeignet, anstatt unter den Betroffenen verteilt zu werden. Umgekehrt, wenn etwa ein demos oder eine Bevölkerungsgruppe ihre delegierten bzw. gewählten AmtsträgerInnen gerade auch aufgrund ihrer narzisstischen Charakterzüge wählt, weil sie besonders gute Leistungen im Dienste der Gemeinschaft versprechen, dann wird das Agieren berechnet, eingeplant und damit explizit in den Dienst politischen Handelns gestellt.

Zwar hat sich bereits Leibniz in der Monadologie dem Problem der unermesslichen Gebiete diffuser, uns selbst unbekannter Bereiche der Vorstellung und Wahrnehmung gestellt, doch erst Freud hat dies mit dem Begriff des Unbewussten und mit seiner "Metapsychologie" systematisiert und empirisch/klinisch für die Behandlung im Einzelsetting wirksam gemacht. Auf der Ebene der Affekte als nicht oder nur schwer durch den Willen "lenkbare" Antriebe gibt es seit der Antike, insbesondere bei Aristoteles, handlungstheoretische Reflexionen, die sich mit den ästhetischen, ethischen und vor allem mit den politischen Implikationen der Affekt- und Triebgesteuertheit befassen. ${ }^{1}$

Anknüpfend an zwei bereits vorliegende Publikationen (Pechriggl 2004 und 2006) möchte ich in der gebotenen Kürze die praxis- und politikphilosophischen Implikationen der Freudschen "Revolution" an der Schnittstelle zwischen der hauptsächlich individuell gefassten Dimension des Agierens und der kollektiv gefassten Dimension des Handelns skizzieren und weiter-

\footnotetext{
${ }^{1}$ Politikphilosophisch relevant sind hier gewiss die aristotelischen Betrachtungen über die politische Rolle der katharsis in der Poetik, die Rhetorik seit Aristoteles, die Marxschen Analysen zur politischen Veränderung insbesondere in seinem Text Der 18. Brumaire des Louis Bonaparte, Webers Charisma-Theorie, Freuds Massenpsychologie und Ich-Analyse unter Bezugnahme auf Le Bon und schließlich, in Anknüpfung an Freud, AutorInnen wie Aulagnier, Kelsen oder Volkan, um nur einige zu nennen.
} 
führen. Unter besonderer Berücksichtigung von Spaltungs- und Dissoziationsmechanismen ist hierfür das Chiasma hilfreich, vermittels dessen auch die herkömmlich entgegen gesetzten Termini der Begriffspaare Individuum-Kollektiv und Agieren-Handeln miteinander verknüpft werden können. Dies ist umso wichtiger, als "Agieren" im psychoanalytischen Sinn ebenso wie Handeln in beiden Registern, dem des Einzelnen und dem des Kollektivs, Sinn machen.

Dazu ist, provisorisch, von Arendts Auffassung abzurücken, dergemäß Handeln nur unter der Bedingung und im Erscheinungsraum der Pluralität Sinn mache (Arendt 1967). Zwar gehe ich gruppenanalytisch und aristotelisch wie Arendt davon aus, dass der Mensch physei ein zôon politikon ist, also eine widersprüchliche Natur hat bzw. ist, d. h. eine "Natur", die vornehmlich darin besteht, sozial, ja politisch, zu sein. Deshalb braucht die Bedingung der Pluralität als Potentialität und als Verwirklichung menschlicher Dispositionen nicht gesondert hinzugefügt zu werden. Doch stellt die Arendtsche Setzung der effektiven Pluralität im öffentlichen "Erscheinungsraum" als notwendige Bedingung für "Handeln" in einer ersten Annäherung für die hier gewählte Begriffssystematik eher ein Hindernis denn eine weiterführende Bestimmung dar. Die Unterscheidung zwischen Agieren und Handeln erfordert vielmehr eine andere Perspektivendifferenzierung, die sowohl auf individueller als auch auf kollektiver Ebene - wenn auch in unterschiedlicher Weise - aktuell wird. Es bedarf der herkömmlichen Perspektive der Intentionalität und der rationalen Planung in Verknüpfung mit Motivation und Affektivität einerseits, der Perspektive der planlosen Blindheit (tychê oder Machiavellis Fortuna) und der zur Desintegration tendierenden Triebsteuerung in Verbindung mit sekundärprozesshafter und immer erst nachträglicher Rationalisierung andererseits.

Nun behält Arendts Betonung der Pluralität als Bedingung für Handeln aber insofern ihre Gültigkeit, als das Spannungsverhältnis zwischen Agieren und Handeln effektiv nur im Erscheinungsraum der Menge als sinnstiftendes bedeutsam ist. Denn das, was im Einzelnen unbewusst, weil verdrängt bzw. abgespalten ist, wird im Kollektiv kaum mit der nämlichen Schärfe und Ausschließlichkeit aus dem Feld des Bewusstseins gedrängt (irgend ein Mensch oder eine Gruppe bringt das "Vergessene" und von vielen oder den meisten Verdrängte mit hoher Wahrscheinlichkeit irgendwann 
explizit und bewusst zum Ausdruck... $)^{2}$. Ich schreibe "kaum", denn es gibt nicht nur kulturelle Tabus sondern auch das, was Foucault l'impensé nennt, das Ungedachte aber auch das Undenkbare oder, was Castoriadis in Anlehnung an die bereits erwähnte Monadologie auf die Gesellschaft ausdehnt und mit dem Begriff der Abgeschlossenheit (clôture) bezeichnet. Dies betrifft aber vorerst nur die eidetische, also die Vorstellungs- und Bedeutungsebene, ohne die wir allerdings kaum etwas über die unbewusste Dimension kollektiver Affekte sagen können. Meine These ist, dass diese Unmöglichkeit kollektiver „Verdrängung“ im starken Sinn ein Kollektiv anfälliger macht für die regressiveren Abwehrformen der projektiven Identitfikation und der Abspaltung.

Was bedeuten aber nun "Abspaltung" und "Verdrängung" bzw. die damit verbundenen projektiven "Mechanismen" auf der Handlungsebene eines Kollektivs und welche sind die Implikationen dieser Phänomene für den Handlungsbegriff? Die vielleicht wichtigste Implikation ist das grundsätzliche Erfordernis, den Handlungsbegriff im Zeichen sowohl psychischer als auch gesellschaftlicher Konflikthaftigkeit zu konzipieren, welche sich gegebenenfalls (aber nicht notwendig) in explizit politischer Konfliktaustragung bzw. -schlichtung artikuliert. Weiters sind für die Differenzierung von Agieren und Handeln folgende begriffliche Spannungsfelder relevant: jenes von aktiv und passiv, von intentionalem Handeln (eines individuellen oder kollektiven Subjekts) und anonymem Passieren/Geschehen/sich Ereignen (es passiert/geschieht/ereignet sich), von gesellschaftlichem Imaginärem (Castoriadis) und Wirklichkeit bzw. Realität sowie von Primärprozess und Sekundärprozess (Freud).

\footnotetext{
${ }^{2}$ Es würde hier zu weit führen, die Zirkularität der Freudschen Instanzen-Metapher (zwischen sozial-politischen und psychischen "Instanzen") genau zu analysieren, sie ist jedenfalls im Sinne einer Rückführung der um die Freudsche (2.) Topik (Ich, Es, Über-Ich) bereicherten Instanzen in das Register des Gesellschaftlichen zu verstehen. Freud entlehnt der rechts- und sozialwissenschaftlichen Terminologie den Instanzenbegriff, um etwas in der Psyche bzw. im Psychischen Geschehen anschaulich zu machen (den Konflikt der Instanzen und die daraus erwachsenden Symptome und Kompromisse). Die Metapher des "Unbewussten der Gesellschaft" (Erheim, Nadig 1982) macht den umgekehrten Sinn explizit: die gesellschaftlichen Kräfte und Schichten, welche analog zum verdrängten Teil des Unbewussten in der Psyche des einzelnen Menschen immer wiederzukehren tendieren. Aber im Unterschied zu den verdrängten Wünschen und Triebvorstellungsrepräsentanzen sind sie bewusste Anteile, deren Verdrängung eine mehr oder weniger offenbare, weil macht- und herrschaftspolitische ist.
} 
Insofern nun aber die Unterscheidung zwischen Agieren und Handeln auf der Ebene des Kollektivs mit der oben beispielhaft illustrierten Spannung zwischen Privat- und Allgemeininteresse bzw. zwischen Trieb/Wunsch des Einzelnen und Sollen der Allgemeinheit verknüpft ist, ja aus dieser hervorzugehen scheint, steht einmal mehr der Konflikt zwischen Einzelnem und Gemeinschaft (oder Allgemeinheit) im Zentrum der Analyse. Dieser Konflikt wurde in der ethisch-politischen Theoriebildung seit der Antike über Kant und Freud bis hin zu Bion auf sehr unterschiedliche Weise diskutiert. Wir können in dieser Tradition der theoretischen Bearbeitung des genannten Konflikts, ja in den einzelnen AutorInnen selbst, zwei Grundtendenzen ausmachen: eine spaltende und eine vermittelnde oder integrierende. Auf der ontischen Ebene, soweit diese von der sie zu ordnen und $\mathrm{zu}$ begreifen suchenden gnoseologischen Ebene überhaupt unterscheidbar ist, sind diese beiden Tendenzen ebenfalls tätig.

Ausgehend von der psychoanalytischen Arbeit mit Gruppen und der diesbezüglichen Theoriebildung (siehe vor allem deren Begründer Bion 1971 und Foulkes 1992) ist nun für den hier behandelten Zusammenhang das rhythmische Oszillieren zwischen antisozial-dissoziativer Tendenz (also dem Hervortreten der egotischen Einzelinteressen) einerseits, verbindend-sozialer Tendenz (Gruppenkohäsion) andererseits, hervorzuheben. Dabei setzt die Integration der Teile, analog zu jener der Partialobjekte, deren Differenzierung auf der Bedeutungsebene, also vermittels einer minimal analysierenden, kategorisierenden und jedenfalls symbolisierenden Sprache voraus. Eine Gruppe oszilliert aber nicht nur zwischen zersetzender und kohäsiv-integrierender Tendenz, sie vermag sich im Zuge kollektiver Regressionen auch in dem einen oder anderen Zustand zu fixieren, also die Rhythmik weitgehend auszusetzen, was zu dauerhafter Abspaltung bzw. Ausgrenzung bestimmter Teile bzw. Anteile der Gruppe führen kann.

Auf der historisch-politischen Betrachtungsebene ist die stasis (Bürgerkrieg, von Zustand, Stillstand) eines der interessantesten Beispiele für eine solche Fixierung unter Beibehaltung der darin entgegen gesetzten Teile, das heißt der "Parteien" oder "Fraktionen". Die Polis im Zu-stand des Bürgerkriegs ist in zwei relativ symmetrische Teile aufgespalten, für die es aktuell keine Vermittlung mehr gibt. Der Kampf beider Teile um die absolute Vorherrschaft umfasst alle Einzelnen, die sich ihm nicht entziehen können (in der stasis nicht Partei zu ergreifen, ist ein Unding, auch für Sklaven und 
Frauen, die ansonsten aus dem Reich des Polemos und der Politik prinzipiell ausgegrenzt sind). Dabei ist die Polis sowohl ideell als auch affektiv gespalten zwischen oligarchischem bzw. tyrannischem Herrschaftsanspruch einerseits, demokratischem Anspruch der Menge auf die Machtausübung andererseits. Alle Affekte und Energien werden in das Austragen dieses Konflikts gelegt, differenzierte Besetzung oder Sublimierung sind nicht mehr möglich, das Regime, also die instituierten Grundformen der Verteilung und Ausübung der Macht stehen vollständig zur Disposition. Die zentrale Frage der Machtausübung bezieht sich vor diesem Hintergrund auf die Dauer bzw. auf die Abwechslung der Machtausübungs sowie auf die Quantität, das heißt darauf, ob die Macht monokratisch (von einem), oligarchisch (von wenigen) oder demokratisch (von der Menge, also dem demos) ausgeübt wird und wie sie in der Zeit verteilt wird bzw. ob sie auf Lebzeiten oder sogar transgenerationell als Herrschaft fixiert ist (auf eine Partei oder ein hereditäres Herrschergeschlecht). Je kleiner und dauerhafter der Kreis der Herrschenden, desto größer muss die Energie, desto effizienter müssen die Dispositive sein, welche die von der Machtausübung Ausgeschlossenen ideologisch, affektiv-sittlich und physisch niederzuringen und auch zukünftig im politischen Abseits zu halten vermögen. Und je weniger ein Demos in einer „Demokratie“ tatsächlich an der institutionellen Machtausübung, also am kratein, beteiligt ist, desto eher werden sich die durch diese Verfehlung der Demokratie aufkommenden Affekte in Form von Aufständen oder anderen krisenhaften Ereignissen Ausdruck verschaffen.

Die Teile des Konflikts sind in der stasis jedoch nicht abgespalten, sondern sie sind als Paroxysmos politischer Opposition einander gegenübergestellt; auch werden die Teile, also die Parteien, nicht immer total, also in der Gesamtheit ihrer Mitglieder, zu vernichten getrachtet, wie dies etwa in ethnischen Bürgerkriegen oder im Zuge von Genoziden der Fall ist bzw. war. Ist die stasis durch den Sieg einer Partei beendet, dann wurde über die Regierungsform entschieden und nicht über die Existenz derer, die von der Macht ausgeschlossen bzw. (im Fall der Oligarchen) um das von ihnen beanspruchte Machtmonopol gekommen sind.

Da im Moment der stasis die rechtliche Formierung der Polis weitgehend außer Kraft gesetzt ist, das heißt, dass sie ohne politeia, ohne Verfassung existiert, wird das Agieren nur noch durch die totale Logik des Ent- 
weder-Oder definiert. Das heißt allerdings nicht, dass die nomoi und die Bestimmungen (graphai) sowie der Ethos in den Menschen nicht irgendwie weiterleben würden oder, dass es überhaupt kein Handeln mehr gäbe. Doch diese Dimensionen des Tuns treten in den Hintergrund und die stasis setzt die Affekte in derart gewaltiger Weise frei, dass sogar die Erinnerung an sie ein Problem darstellt: Die historiographische und politischphilosophische Analyse der stasis oder des Bürgerkriegs stößt regelmäßig auf das Phänomen der Ausblendung, der Tabuisierung und der Verleugnung (Loraux 1997).

\section{Acting out versus deliberation}

Wie Leibniz in der Monadologie hervorhob, ist der Großteil unserer perceptions (sinnliche und/oder intelligible Wahrnehmungen, Vorstellungen, Gedanken, etc.) uns nicht klar bewusst, sondern sie bleiben diffus miteinander vermengt. Dennoch sind diese "unbewussten" Vorstellungen massiv wirksam, also effektiv im aristotelischen Sinn. Erst die begrifflich geleitete Analyse der Details, also ihre Herauslösung aus der Amorphheit des psychosomatischen Gemischs aus Sinnesreizen, -wahrnehmungen, eindrücken, Grundannahmen etc. erlauben eine gewisse, immer durch unsere anthropologischen Bedingtheiten beschränkte, Klärung oder eine gewisse Selbstaufklärung der Psyche und der Gesellschaft als aus vielen Psychen zusammengesetzte.

Die Analyse ist aber vom ontischen Gesichtspunkt her betrachtet zugleich ein Verfahren, das zur Auflösung eines Zustands affektiver Aufgelöstheit führt. Politisches Handeln im Sinne expliziter Beratung und Entscheidung setzt voraus, dass die sich im Zustand der Auflösung (alytos) befindliche Gruppe (oder Gesellschaft) die affektive wie auch ideelle Diffusität zu unterteilen und aufzugliedern vermag und, dass sie sich zugleich - nach Maßgabe der Möglichkeiten - auf solche Formen und Verfahrensweisen "einigt", die der damit einhergehenden Analyse, Beratung und Entscheidung förderlich sind, was ja einen Sinnkern des Nomos, dann des gesatzten Rechts ausmacht. Es kann aber, wie vornehmlich Kelsen dargelegt hat, nicht ohne das implizite oder explizite Postulat einer göttlichen Ordnungsinstanz eine allgemeingültige Grundform angenommen werden, die jenseits der gesetzten und instituierten Formen und Normen der Gliede- 
rung, Systematisierung und Machtverteilung angesiedelt wäre. Nachvollziehbarer und weniger surreal ist dagegen die Annahme, dass Normierung aus der kollektiven Bereitschaft und Fähigkeit hervorgeht, das erwähnte Oszillieren selbst mit Hilfe der Analyse und vermittels des Handelns und der Gesetzgebung zu gestalten und nicht alles dem passierenden Agieren und einigen mehr oder weniger ,repräsentativen“ Führern eines solchen kollektiven Agierens zu überlassen. Doch umgekehrt kippt die Hypertrophie des planend rationalisierenden Handelns rasch in den Modus des Sozialengineerings, auf den die Wiederkehr des "Verdrängten", des aus dem planend-diskursiven Verfügen Ausgegrenzten und davon Abgespaltenen, folgt.

Nun machen planendes und analytisches Handeln nicht immer Sinn. Wo hinreichende Informationen und/oder Übersicht über die Lage nicht gegeben sind, bedarf es der Intuition, der enthymemata wie Aristoteles diese Art von Vorstellungen nennt. Dies gilt vor allem auf der persönlichexistentiellen Ebene, ebenso auf der weniger formalisierten bzw. formalisierbaren Ebene des Zusammenlebens, wo die Einbeziehung und Abwägung der unterschiedlichen Intuitionen von größter ethischer wie auch politischer Bedeutung ist (eine Nacht über eine Entscheidung schlafen - ein Gebot, das für triftige Entscheidungen sogar in der antiken ekklesia galt, etwa als es darum ging, die Milesier schon oder nicht zu töten).

Völlige Auflösung im (Aus)Agieren (etwa einer Panik) ist ein ebenso unwahrscheinliches Phänomen wie totale Planung: Das Chaotische ist immer mit Planung durchsetzt, die Planung immer mit Unberechenbarem. Im Falle der Trennung können die beiden Extreme rasch in ihr Gegenteil kippen, denn die dauerhafte Trennung beider ist für das Zusammenleben ebenso problematisch wie die Auflösung der Handlungsdimension in jener des Agierens. Abspaltung und Fixierung sind dann die Ursache für eine Entkoppelung des Imaginären von der Wirklichkeit oder umgekehrt: des verwaltenden Realitätsprinzips von der schöpferischen Phantasie. Erstere mündet in der blindwütigen Vernichtung der im kollektiven Wahn errichteten Feinde, Letztere in der technizistisch-bürokratischen Vorherrschaft des Funktionalen. Der Nationalsozialismus etwa schaffte einen Kurzschluss beider: Er erfand nicht nur sondern realisierte - mit einer zuvor nicht da gewesenen Effizienz - die industrielle Vernichtung des wahnhaften Feindes namens "Juden". Dieses Phänomen zu integrieren erfordert eine 
größtmögliche Variationsbreite zwischen Analyse, Intuition, besonnenem Handeln und grenzgängerischem Agieren (gegen eine verleugnende Staatsräson etwa), welche die Matrix für weitere Auseinandersetzungen bilden. Im vermittelten Modus geht das Agieren in den Prozess der Normsetzung um einer "rechten" Normsetzung willen ein und wird somit nicht zum ausschließlichen Motor und Zweck der Selben.

\section{LITERATUR}

Arendt, H. 1967 Vita Activa, München: Piper.

Bion, W. 1971 Erfahrungen in Gruppen, Stuttgart: Klett-Cotta.

Castoriadis, C. 1984 Die Gesellschaft als imaginäre Institution, Frankfurt/Main: Suhrkamp.

Erdheim, M. und Nadig, M. 1982 Die gesellschaftliche Produktion von Unbewußtheit, Frankfurt/M.: Suhrkamp.

Foulkes, S.H. 1992 Gruppenanalytische Psychotherapie, München: Pfeiffer.

Leibniz 1970 Monadologie, Paris: Delagrave.

Loraux, N. 1997 La cité divisée. L'oubli dans la mémoire d'Athenes, Paris: Payot.

Pechriggl, A. 2004 "'Biopolitik' und die Grenzen demokratischer Instituierung zwischen Agieren und Handeln" in: Frankfurter Arbeitskreis für politische Theorie \& Philosophie (Hg.), Autonomie und Heteronomie der Politik. Politisches Denken zwischen Post-Marxismus und Proststrukturalismus, Bielefeld: Transcript, 33-55.

- 2006 "Politik der Angst zwischen Agieren und Handeln", in Politiken der Angst, AUF eine Frauenzeitschrift, 131, 4-9. 
